\title{
INFLUÊNCIA DO pH NA ADSORÇÃO DE ÍONS Cr (VI) UTILIZANDO ESPONJA DE QUITOSANA
}

\author{
E. L. ARAÚJO' ${ }^{1}$, C.E. G.COMIOTTO ${ }^{1}$, M. A. LOPES$^{1}$,T. R. S. CADAVAL Jr ${ }^{1}$ e L. A. de A. \\ PINTO $^{1}$
}

${ }^{1}$ Universidade Federal do Rio Grande, Escola de Química e Alimentos

E-mail para contato: dqmpinto@furg.br

\begin{abstract}
RESUMO - Neste trabalho, o efeito do pH (faixa de 2 a 8) foi estudado na adsorção de íons Cr (VI) utilizando esponja de quitosana, e analisadas as possíveis interações entre a esponja e o metal, através das técnicas de infravermelho com transformada de Fourier (FT-IR) e calorimetria exploratória diferencial (DSC). A capacidade de adsorção (q) atingiu o seu valor máximo em $\mathrm{pH} 6$, o qual foi de $213,4 \mathrm{mg} / \mathrm{g}$. A partir dos espectros vibracionais, antes e após a adsorção, foram identificadas alterações nas regiões dos amino grupos e das hidroxilas da esponja de quitosana.Com base na análise de DSC, foi observado que a presença de $\mathrm{Cr}$ (VI) após a adsorção provocou alterações no comportamento estrutural da esponja.
\end{abstract}

\section{INTRODUÇÃO}

O cromo hexavalente $\mathrm{Cr}$ (VI) é metal que está presente em grande quantidade nos efluentes de atividades como curtumes, galvanoplastia e mineração, os quais devem ser tratados devido a sua alta toxicidade (Cadaval Jr et al., 2013). Métodos convencionais de tratamento como filtração, precipitação química, resinas de troca iônica, eletrodeposição e membranas são desfavoráveis do ponto de vista econômico e/ou operacional (Ali et al., 2012). Nesse contexto, a adsorção se apresenta como uma alternativa viável para a remoção de $\mathrm{Cr}$ (VI) de efluentes líquidos devido a sua simplicidade e relação custo benefício.

Atualmente, diversos adsorventes de fontes renováveis têm sido estudados, dentre os quais a quitosana pode ser destacada. Entretanto, na forma de pó, a quitosana apresenta desvantagens como pequena área superficial e baixa porosidade (Kannusamye Sivalingam, 2013). Estas limitações podem ser contornadas por meio da obtenção de esponjas de quitosana, as quais apresentam uma elevada área superficial e alta porosidade, facilitando a transferência de massa por convecção e difusão intrapartícula.

Dentre os parâmetros que influenciam na capacidade de adsorção de materiais a base de quitosana, o pH é um dos mais importantes. Sendo assim, o objetivo deste trabalho foi estudar a influência do pH na adsorção de íons $\mathrm{Cr}$ (VI) utilizando esponja de quitosana, bem como a caracterização das mesmas antes e após a adsorção utilizando as técnicas de infravermelho com transformada de Fourier (FT-IR) e calorimetria exploratória diferencial (DSC). 


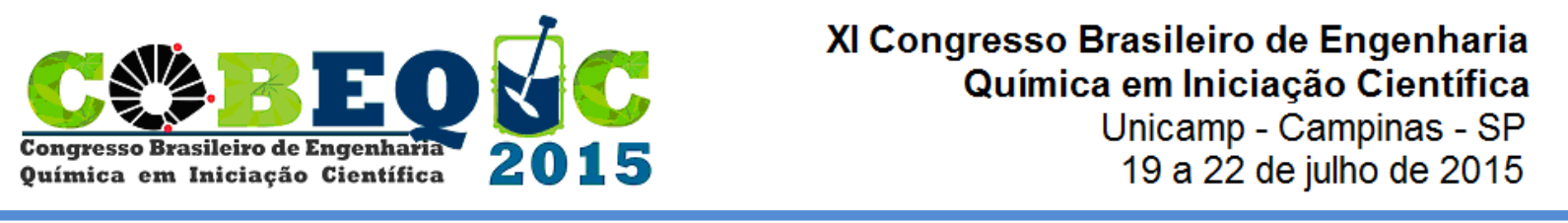

\section{MATERIAL E MÉTODOS}

\subsection{Preparação da Esponja de Quitosana}

Quitosana em pó (massa molar $150 \pm 3 \mathrm{kDa}$, grau de desacetilação de $85 \pm 1 \%$ e diâmetro médio de $72 \pm 3 \mu \mathrm{m}$ ) foi obtida de resíduos de camarão (Penaeus brasiliensis) (Weska et al., 2007). Uma massa de $2 \mathrm{~g}$ foi dissolvida em $100 \mathrm{~mL}$ de solução de ácido acético $1,0 \%(\mathrm{~m} / \mathrm{v})$, e após a solução foi homogeneizada à $10.000 \mathrm{rpm}$ por $5 \mathrm{~min}$ em um agitador mecânico (Dremel, 1100-01, Brasil), e levada a um ultra freezer (Indrel, IULT 90-D, Brasil) por $48 \mathrm{~h}$ a $-80^{\circ} \mathrm{C}$. Finalmente, o material foi submetido à secagem a frio em um liofilizador (Liobras, L108, Brasil) por $48 \mathrm{~h}$ com vácuo de $44 \mathrm{mmHg}$ e temperatura de $-54^{\circ} \mathrm{C}$. A amostra foi colocada em um dessecador para posterior uso (Landi et al., 2008).

\subsection{Experimentos de Adsorção}

Foi preparada uma solução estoque de íons de $\mathrm{Cr}$ (VI) $(1,0 \mathrm{~g} / \mathrm{L})$ utilizando $\mathrm{K}_{2} \mathrm{Cr}_{2} \mathrm{O}_{7}$ (99,0\% Merck, Alemanha) e água destilada. A partir da solução estoque foram preparadas soluções com concentração de $100 \mathrm{mg} / \mathrm{L}$ sob diferentes condições de $\mathrm{pH}(2,4,6,8)$. O pH foi ajustado utilizando solução tampão fosfato dissódico $0,1 \mathrm{~mol} / \mathrm{L}$ e ácido cítrico $0,1 \mathrm{~mol} / \mathrm{L}$. Os experimentos foram então realizados adicionando-se as esponjas de quitosana nas soluções preparadas. Estas soluções foram agitadas (Nova Ética, 109-1TCM, Brasil) a $100 \mathrm{rpm}$ e temperatura de $25{ }^{\circ} \mathrm{C}$ até o equilíbrio. A concentração de íons de $\mathrm{Cr}$ (VI) remanescente na fase líquida foi determinada por espectroscopia de absorção atômica de chama (GBC Avanta, 932AA, Austrália). Todos os experimentos foram realizados em réplica $(n=3)$. A capacidade de adsorção foi determinada pela Equação 1.

$$
q=\left[\frac{\left(C_{0}-C_{e}\right)}{m}\right] V
$$

Sendo $C_{0}$ e $C_{e}$, respectivamente, as concentrações inicial e de equilíbrio na fase líquida $(\mathrm{mg} / \mathrm{L}), m$ a massa de adsorvente (g) e $V$ o volume da solução (L).

\subsection{Análise das Interações}

As interações entre adsorvente e adsorbato foram elucidadas utilizando um espectroscópio infravermelho com transformada de Fourier (FT-IR) (Prestige, 21210045, Japão) e calorimetria exploratória diferencial (DSC) (DSC-60, Shimadzu). As esponjas de quitosana foram analisadas antes e após a operação de adsorção (Esquerdo et al., 2014).

\section{RESULTADOS E DISCUSSÃO}

\subsection{Efeito do pH}

O efeito do pH na capacidade de adsorção $(q)$ de íons de $\mathrm{Cr}$ (VI) utilizando esponja de quitosana está apresentado na Figura 1. 
Figura 1 - Efeito do pH na capacidade de adsorção de íons de Cr (VI) utilizando esponjas de quitosana como adsorvente.

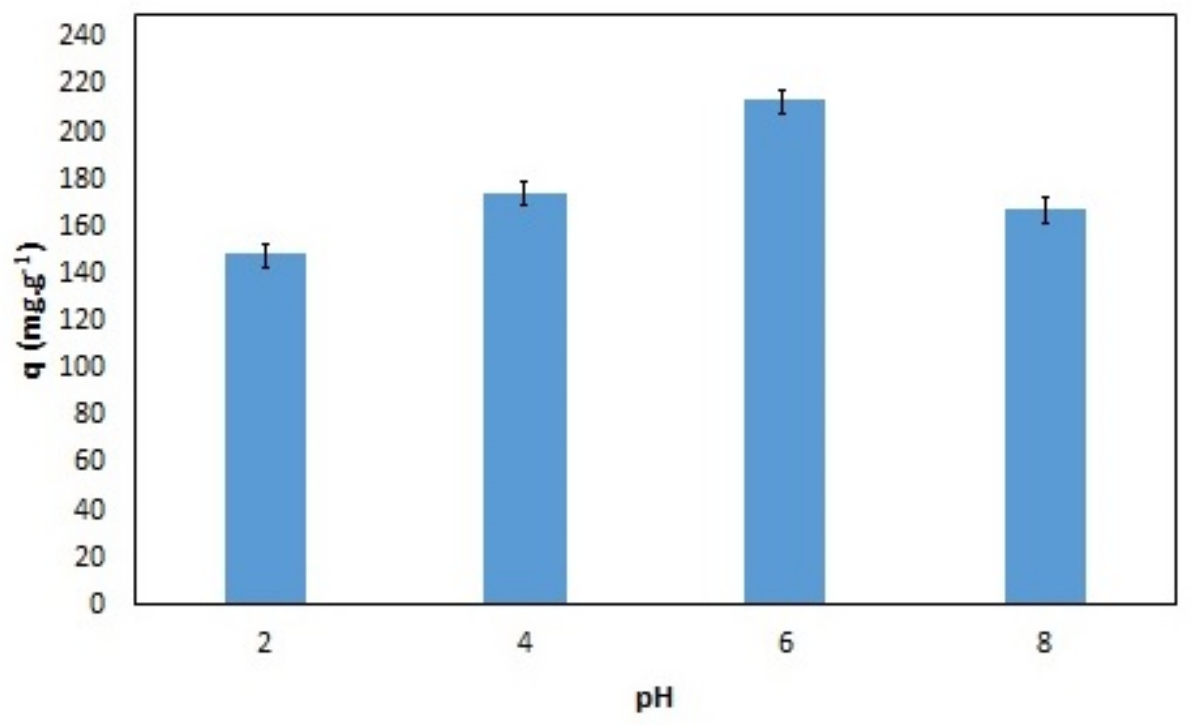

Conforme mostra a Figura 1, é possível observar que a capacidade de adsorção da esponja de quitosana foi superior em $\mathrm{pH} 6$, sendo seu valor de $213,4 \mathrm{mg} / \mathrm{g}$. Estudos realizados com a quitosana em pó na adsorção de $\mathrm{Cr}$ (VI) em condições semelhantes às deste trabalho tiveram valores de capacidade de adsorção máximo de 97,4 mg/g (Cadaval Jr et al., 2013). É possível observar também que mesmo nas demais condições de $\mathrm{pH}$ estudadas, a capacidade de adsorção da esponja foi consideravelmente superior à da referida quitosana em pó, demonstrando a influência do aumento da área superficial e da porosidade no aumento da capacidade de adsorção do material.

\subsection{Interações}

Análise de FT-IR: As interações entre a esponja de quitosana e os íons de $\mathrm{Cr}$ (VI) foram estudadas utilizando a técnica de espectroscopia de infravermelho com transforma de Fourier (FT-IR). As Figuras 2 (a) e 2 (b) mostram respectivamente o espectro vibracional da esponja antes e após a operação de adsorção. A Figura 2 (a) apresenta as bandas características da esponja de quitosana relativas aos estiramentos axiais das ligações $\mathrm{N}-\mathrm{H}$ e $\mathrm{O}-\mathrm{H}$ entre 3100 e $3500 \mathrm{~cm}^{-1}$ e os estiramentos axiais das ligações $\mathrm{C}-\mathrm{N}$ em 1550 e $1075 \mathrm{~cm}^{-1}$. Podemos identificar que após a adsorção (Figura 2 (b)) ocorreram alterações na região dos grupamentos relativos à amina e a hidroxila das moléculas de quitosana. Isto indica a interação entre as aminas e as hidroxilas da esponja de quitosana com os íons de $\mathrm{Cr}$ (VI).

Figura 2 - Espectro de infravermelho da esponja de quitosana: (a) antes da adsorção; (b) após a adsorção. 

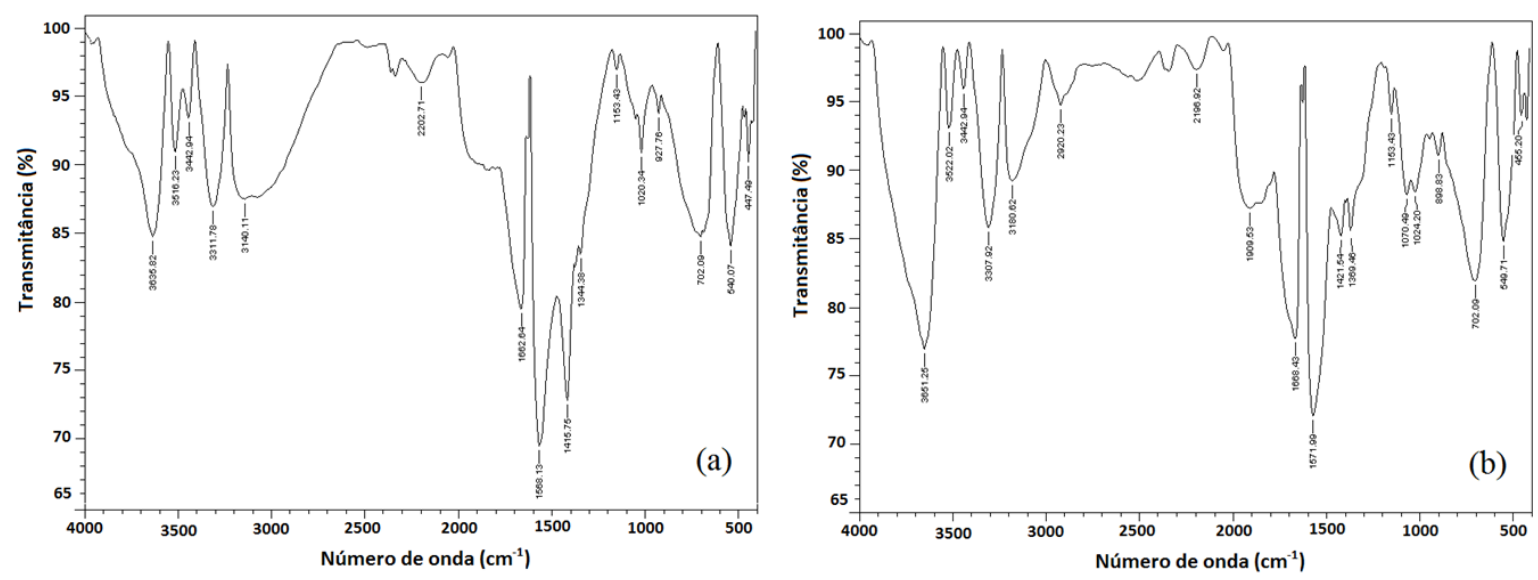

Análise de DSC: Conforme mostra a Figura 3, um pico endotérmico por volta de $100{ }^{\circ} \mathrm{C}$ foi observado em ambas às curvas. Estes picos são atribuídos à evaporação da água residual. Além disso, um pico exotérmico por volta de $275^{\circ} \mathrm{C}$ foi observado apenas na curva referente à esponja antes da adsorção, o qual pode ser devido a degradação da cadeia polimérica da quitosana (Rodrigues et al., 2009). Entretanto na curva relativa à esponja, após a adsorção, este mesmo pico não ocorre. Esta diferença no comportamento pode ser atribuída a presença dos íons de Cr (VI) na amostra, os quais podem ter alterado as interações existentes na esponja pura, inibindo sua degradação.

Figura 3 - Curvas de DSC da esponja de quitosana antes e após a adsorção.

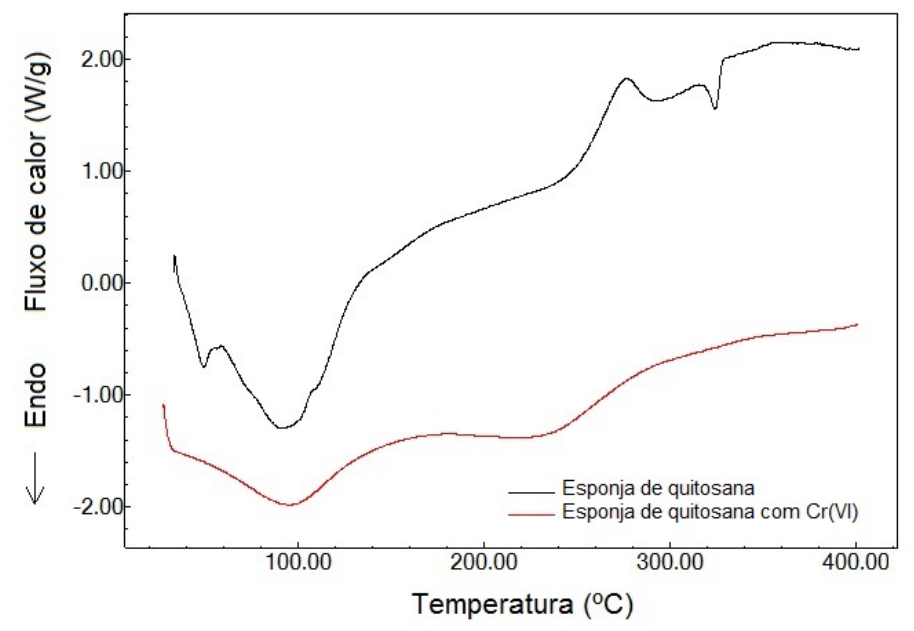

\section{CONCLUSÃO}


Neste trabalho, verificou-se a influência do pH na adsorção de íons de Cr (VI) por esponja de quitosana e foram elucidadas as interações esponja-íons de $\mathrm{Cr}$ (VI). Na faixa de pH estudada, a capacidade de adsorção (q) apresentou seu máximo em $\mathrm{pH}$ 6, com valor de $213,4 \mathrm{mg} / \mathrm{g}$. Através da análise de FT-IR foi verificada a preferência dos íons de Cr (VI) pelos grupamentos amina e hidroxila presentes na esponja de quitosana. A análise de DSC indicou que a presença de íons Cr (VI) adsorvidos, inibe as alterações estruturais da esponja de quitosana ocorridas antes da adsorção.

\section{REFERÊNCIAS}

ALI, I.; ASIM, M.; KHAN, T. A. Low cost adsorbents for the removal of organic pollutants from wastewater.J. Environ. Manage., v. 113, p. 170-183, 2012.

CADAVAL Jr, T. R. S.; CAMARA, A. S.; DOTTO, G. L.; PINTO, L. A. A. Adsorption of Cr (VI) by chitosan with different deacetylation degrees. Desalin. Water Treat.,v. 51, p. 76907699, 2013.

ESQUERDO, V. M.; CADAVAL Jr, T. R. S.; DOTTO, G. L.; PINTO, L. A. A. Chitosan scaffold as an alternative adsorbent for the removal of hazardous food dyes from aqueous solutions. J. Colloid Interface Sci., v. 424, p. 7-15, 2014.

KANNUSAMY, P.; SIVALINGAM, T.Synthesis of porous chitosan-polyaniline/ZnO hybrid composite and application for removal of reactive orange 16 dye. Colloids Surf., B,v. 108, p. 229-238, 2013.

LANDI, E.; VALENTINI, F; TAMPIERI, A. Porous hydroxyapatite/gelatine scaffolds with ice-designed channel-like porosity for biomedical applications. Acta Biomater.,v. 4, p. 1620$1626,2008$.

RODRIGUES, L. B.; LEITE, H. F.; YOSHIDA, M. I.; SALIBA J. B.; CUNHA Jr., A. S.; FARACO, A. A. G. In vitro release characterization of chitosan films as dexamethasone carrier. Int. J. Pharm., v. 368, p. 1-6, 2009.

WESKA, R. F.; MOURA, J. M.; BATISTA, L. M.,; RIZZI, J.; PINTO, L. A. A. Optimization of deacetylation in the production of chitosan from shrimp wastes: Use of response surface methodology. J. Food Eng., v. 80, p. 749-753, 2007. 